\title{
Tromboflebitis de la vena toracoepigástrica: enfermedad de Mondor
}

\author{
Thoracoepigastric vein thrombophlebitis: Mondor's disease \\ Luis Gerardo Domínguez Carrillo, * Luis Gerardo Domínguez Gasca ${ }^{\ddagger}$
}

Citar como: Domínguez CLG, Domínguez GLG. Tromboflebitis de la vena toracoepigástrica: enfermedad de Mondor. Acta Med. 2020; 18 (4): 429-430. https://dx.doi.org/10.35366/97275

Masculino de 30 años, profesionista, deportista, sin antecedentes de importancia, presenta dolor torácico localizado sobre la sexta articulación condrocostal izquierda de dos días de evolución. Acude a traumatólogo quien diagnostica síndrome de Tietze y lo deriva a fisioterapia. A la exploración en rehabilitación 24 horas después se observa piel de la zona sin cambios de coloración, línea de elevación subcutánea de $10 \mathrm{~cm}$ de longitud partiendo de la sexta articulación costocondral izquierda hasta el borde costal, que corresponde a cordón indurado a la palpación, sensible a la presión en su parte proximal (Figura 1); el dolor se incrementa con contracción de músculos abdominales y con elevación de extremidad torácica izquierda. Con diagnóstico de enfermedad de Mondor se solicita ultrasonido Doppler de la vena toracoepigástrica, el cual confirma el diagnóstico (Figura 2). Se prescriben antiinflamatorios no esteroideos por 14 días. En el seguimiento a 30 días, paciente asintomático sin datos clínicos de induración de la vena toracoepigástrica.

La tromboflebitis superficial de la vena toracoepigástri$\mathrm{ca}^{1,2}$ o enfermedad de Mondor fue mencionada inicialmente por Faage en 1869 y posteriormente por Henry Mondor en 1939, quien la describió histológicamente como una "angeítis subcutánea". Presenta relación de géneros a favor del femenino 9:1 a 14:1; en hombres se calcula incidencia de $1.39 \%$ entre 20 y 40 años de edad, sus causas son: idiopática en $45 \%$ de los casos; $20 \%$ iatrogénica (cirugía de tórax, radiación y terapia hormonal); $22 \%$ traumática (incluyendo ejercicio intenso) y 5\% asociado a cáncer de mama.

Históricamente la "tríada de Vichow" caracterizada por lesión vascular, estasis sanguínea e hipercoagulabilidad y descrita hace más de 150 años continúa vigente, agregándose sólo mayor fineza, siendo: a) lesión en la pared vascular, anomalías en el endotelio tales como aterosclerosis e inflamación vascular asociada; b) estasis circulatoria:

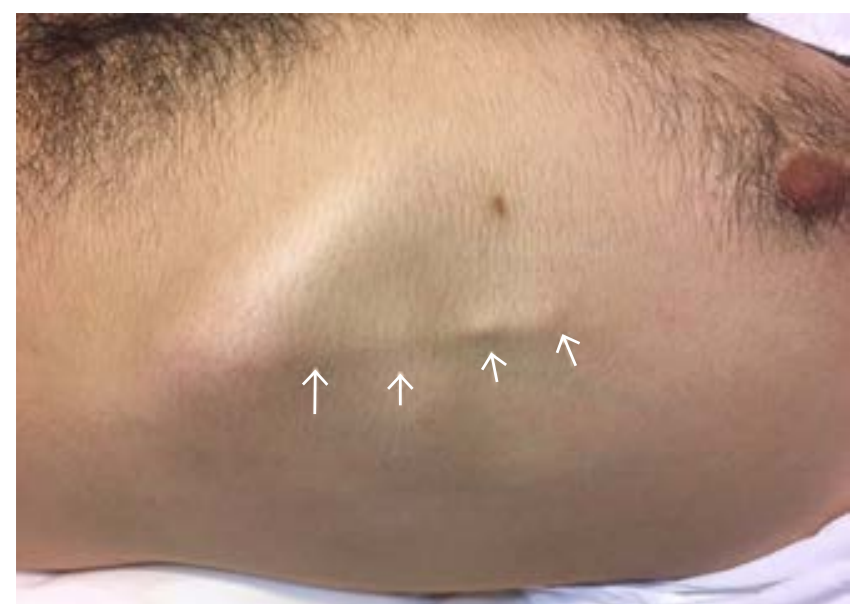

Figura 1: Fotografía clínica que muestra hemitórax izquierdo de masculino de 30 años, con elevación subcutánea concerniente a cordón indurando de $10 \mathrm{~cm}$ de longitud (flechas), que corresponde a tromboflebitis de la vena toracoepigástrica izquierda. (Enfermedad de Mondor).
* Especialista en Medicina de Rehabilitación. Catedrático de la Facultad de Medicina de León. Universidad de Guanajuato.

‡ Ortopedista. División de Cirugía del Hospital Ángeles León. León, Guanajuato. México.
Correspondencia:

Dr. Luis Gerardo Domínguez Carrillo

Correo electrónico: Igdominguez@hotmail.com

Aceptado: 08-11-2019.

www.medigraphic.com/actamedica

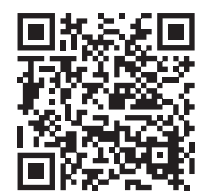



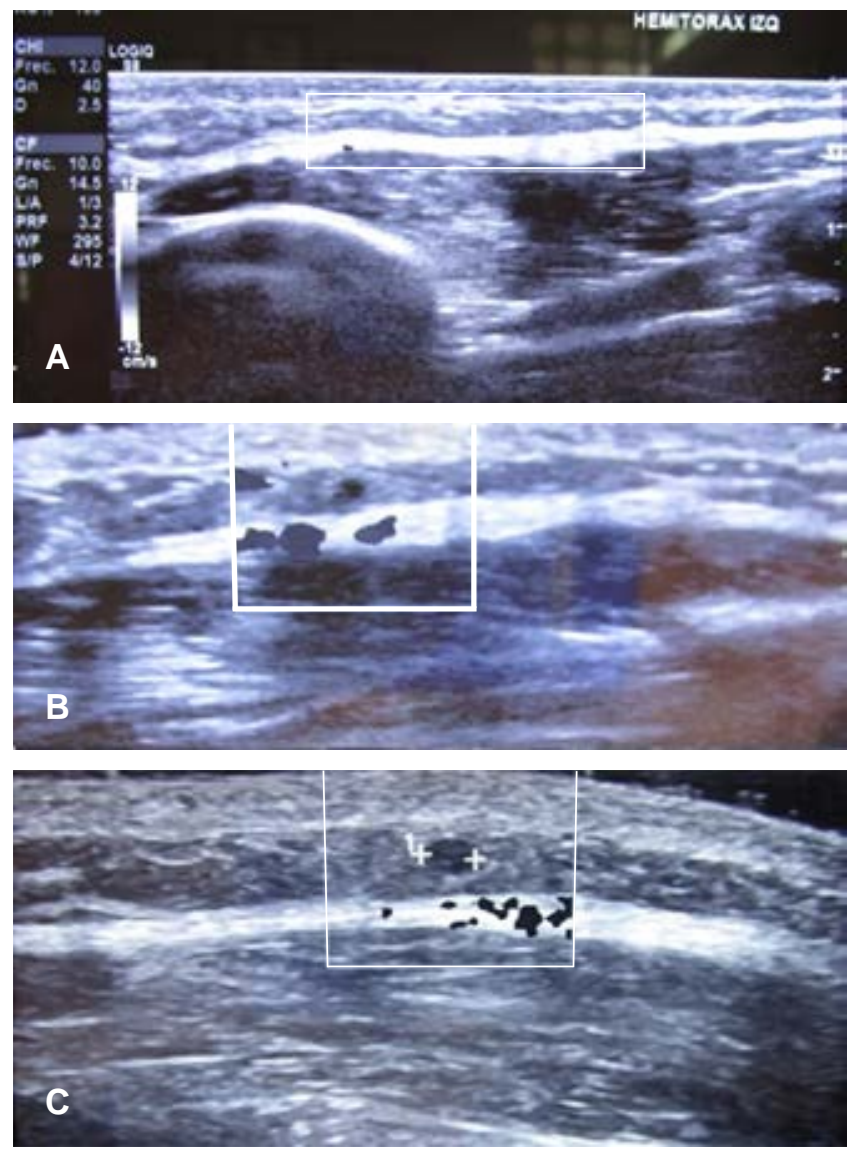

Figura 2: Estudio de ultrasonografía. A) Corte sagital. B) Doppler (color negativo). C) Corte transversal. Se muestra trayecto vascular subcutáneo inflamatorio de $7 \mathrm{~cm}$ de longitud y $4 \mathrm{~mm}$ de diámetro, no se modifica con maniobras de compresión y corresponde a tromboflebitis de vena superficial de hemitórax izquierdo. (Enfermedad de Mondor).

anomalías hemorreológicas y turbulencia en las bifurcaciones vasculares y en las regiones vasculares estenóticas; y c) estado de hipercoagulabilidad: anomalías en las vías de coagulación y fibrinolíticas y en la función plaquetaria asociadas a mayor riesgo de TEV y otras cardiovasculopa- tías como arteriopatía coronaria, insuficiencia cardiaca y apoplejía en pacientes con fibrilación auricular.

Clínicamente se manifiesta como dolor localizado en la pared torácica, a la palpación un cordón subcutáneo indurado en el trayecto de la vena toracoepigástrica con predominio del lado izquierdo, sin cambios inflamatorios en la piel. ${ }^{3}$

Histológicamente, de manera inicial aparece infiltrado inflamatorio en la pared del vaso con trombo que ocluye la luz, lo que posteriormente origina proliferación de tejido conectivo que resulta en la formación de un cordón indurado. Con inmunohistoquímica se detecta positividad para anticuerpos monoclonales CD31 y CD34 indicando origen venoso de la lesión. ${ }^{4}$

La ecografía es la elección para corroborar diagnóstico, se identifica el trayecto del vaso superficial afectado que muestra una estructura tubular alargada, anecoica y sin flujo en el Doppler. ${ }^{5}$

El diagnóstico diferencial debe realizarse con desgarros musculares, síndrome de Tietze, infección por larva migrans y granuloma intersticial por colagenopatía.

Respecto al tratamiento, dado que la lesión tiende a la resolución espontánea y es autolimitada, su manejo es conservador con antiinflamatorios no esteroideos; en casos de dolor severo se puede recurrir a la infiltración local de anestésico, siendo excepcional la trombectomía o resección venosa superficial.

\section{REFERENCIAS}

1. Hernández CCM, Pérez NV, Guerrero $A O$, Rodríguez $C N$, Fonseca RJC. Superficial thrombophlebitis of the thoracoepigastric vein: Mondor's disease. Dermatología Cosmética, Médica y Quirúrgica. 2018; 16: 157-160.

2. Uribe G, Sigler L, Gutiérrez CR, Sánchez FC, Jiménez GR. Tromboflebitis toracoabdominal. Rev Mex Angiol. 2012; 40: 57-60.

3. Masayuki A, Taro S. Mondor's disease: a review of the literature. Intern Med. 2018; 57: 2607-2612.

4. Ichinose A, Fukunaga A, Terashi H. Objective recognition of vascular lesions in Mondor's disease by immunohistochemistry. J Eur Acad Dermatol Venereol. 2008; 22: 168-173.

5. Yanik B, Conkbayir I, Oner O, Hekimoğlu B. Imaging findings in Mondor's disease. J Clin Ultrasound. 2003; 31: 103-107. 